\title{
Search for heavy neutral leptons decaying into muon-pion pairs in the MicroBooNE detector
}

P. Abratenko ${ }^{35}$ M. Alrashed, ${ }^{15}$ R. An,${ }^{14}$ J. Anthony ${ }^{4}$ J. Asaadi,${ }^{34}$ A. Ashkenazi, ${ }^{19}$ S. Balasubramanian, ${ }^{38}$ B. Baller,${ }^{11}$ C. Barnes, ${ }^{20}$ G. Barr, ${ }^{24}$ V. Basque, ${ }^{18}$ S. Berkman, ${ }^{11}$ A. Bhanderi, ${ }^{18}$ A. Bhat,${ }^{31}$ M. Bishai, ${ }^{2}$ A. Blake, ${ }^{16}$ T. Bolton, ${ }^{15}$ L. Camilleri, ${ }^{9}$ D. Caratelli, ${ }^{11}$ I. Caro Terrazas, ${ }^{8}$ R. Castillo Fernandez,${ }^{11}$ F. Cavanna,${ }^{11}$ G. Cerati, ${ }^{11}$ Y. Chen, ${ }^{1}$ E. Church,${ }^{25}$ D. Cianci, ${ }^{9}$ E. O. Cohen, ${ }^{32}$ J. M. Conrad, ${ }^{19}$ M. Convery, ${ }^{29}$ L. Cooper-Troendle, ${ }^{38}$ J. I. Crespo-Anadón, ${ }^{9}$ M. Del Tutto, ${ }^{13,11}$ A. Devitt, ${ }^{16}$ L. Domine, ${ }^{29}$ K. Duffy, ${ }^{11}$ S. Dytman, ${ }^{26}$ B. Eberly, ${ }^{10}$ A. Ereditato, ${ }^{1}$ L. Escudero Sanchez, ${ }^{4}$ J. J. Evans, ${ }^{18}$ R. S. Fitzpatrick, ${ }^{20}$ B. T. Fleming ${ }^{38}$ N. Foppiani, ${ }^{13}$ D. Franco, ${ }^{38}$ A. P. Furmanski, ${ }^{18,21}$ D. Garcia-Gamez, ${ }^{12}$ S. Gardiner, ${ }^{11}$ V. Genty, ${ }^{9}$ D. Goeldi, ${ }^{1}$ S. Gollapinni, ${ }^{33,17}$ O. Goodwin, ${ }^{18}$ E. Gramellini, ${ }^{11}$ P. Green, ${ }^{18}$ H. Greenlee, ${ }^{11}$ L. Gu,${ }^{36}$ W. Gu, ${ }^{2}$ R. Guenette, ${ }^{13}$ P. Guzowski, ${ }^{18}$ P. Hamilton, ${ }^{31}$ O. Hen, ${ }^{19}$ C. Hill, ${ }^{18}$ G. A. Horton-Smith, ${ }^{15}$ A. Hourlier, ${ }^{19}$ E.-C. Huang, ${ }^{17}$ R. Itay, ${ }^{29}$ C. James, ${ }^{11}$ J. Jan de Vries, ${ }^{4}$ X. Ji, ${ }^{2}$ L. Jiang, ${ }^{26,36}$ J. H. Jo, ${ }^{38}$ R. A. Johnson, ${ }^{7}$ J. Joshi, ${ }^{2}$ Y.-J. Jwa, ${ }^{9}$ G. Karagiorgi, ${ }^{9}$ W. Ketchum, ${ }^{11}$ B. Kirby, ${ }^{2}$ M. Kirby, ${ }^{11}$ T. Kobilarcik, ${ }^{11}$ I. Kreslo, ${ }^{1}$ R. LaZur, ${ }^{8}$ I. Lepetic,${ }^{14}$ Y. Li ${ }^{2}$ A. Lister, ${ }^{16}$

B. R. Littlejohn, ${ }^{14}$ S. Lockwitz, ${ }^{11}$ D. Lorca, ${ }^{1}$ W. C. Louis, ${ }^{17}$ M. Luethi, ${ }^{1}$ B. Lundberg, ${ }^{11}$ X. Luo, ${ }^{38,3}$ A. Marchionni, ${ }^{11}$ S. Marcocci, ${ }^{11}$ C. Mariani, ${ }^{36}$ J. Marshall, ${ }^{37}$ J. Martin-Albo, ${ }^{13}$ D. A. Martinez Caicedo, ${ }^{30}$ K. Mason, ${ }^{35}$ A. Mastbaum, ${ }^{6,27}$ N. McConkey ${ }^{18}$ V. Meddage ${ }^{15}$ T. Mettler, ${ }^{1}$ K. Miller, ${ }^{6}$ J. Mills, ${ }^{35}$ K. Mistry, ${ }^{18}$ A. Mogan, ${ }^{33}$ T. Mohayai, ${ }^{11}$ J. Moon, ${ }^{19}$ M. Mooney, ${ }^{8}$ C. D. Moore, ${ }^{11}$ J. Mousseau, ${ }^{20}$ R. Murrells, ${ }^{18}$ D. Naples, ${ }^{26}$ R. K. Neely, ${ }^{15}$ P. Nienaber, ${ }^{28}$ J. Nowak, ${ }^{16}$

O. Palamara, ${ }^{11}$ V. Pandey, ${ }^{36}$ V. Paolone, ${ }^{26}$ A. Papadopoulou, ${ }^{19}$ V. Papavassiliou, ${ }^{22}$ S. F. Pate, ${ }^{22}$ A. Paudel, ${ }^{15}$ Z. Pavlovic,${ }^{11}$

E. Piasetzky, ${ }^{32}$ D. Porzio $\odot,{ }^{18}$ S. Prince,${ }^{13}$ G. Pulliam, ${ }^{31}$ X. Qian, ${ }^{2}$ J. L. Raaf, ${ }^{11}$ V. Radeka, ${ }^{2}$ A. Rafique,${ }^{15}$ L. Ren, ${ }^{22}$ L. Rochester, ${ }^{29}$ H. E. Rogers, ${ }^{8,5}$ M. Ross-Lonergan, ${ }^{9}$ C. Rudolf von Rohr, ${ }^{1}$ B. Russell, ${ }^{38}$ G. Scanavini, ${ }^{38}$ D. W. Schmitz ${ }^{6}$ A. Schukraft, ${ }^{11}$ W. Seligman, ${ }^{9}$ M. H. Shaevitz, ${ }^{9}$ R. Sharankova, ${ }^{35}$ J. Sinclair, ${ }^{1}$ A. Smith, ${ }^{4}$ E. L. Snider, ${ }^{11}$ M. Soderberg, ${ }^{31}$ S. Söldner-Rembold, ${ }^{18}$ S. R. Soleti, ${ }^{24,13}$ P. Spentzouris, ${ }^{11}$ J. Spitz, ${ }^{20}$ M. Stancari, ${ }^{11}$ J. St. John, ${ }^{11}$ T. Strauss, ${ }^{11}$ K. Sutton, ${ }^{9}$ S. Sword-Fehlberg, ${ }^{22}$ A. M. Szelc, ${ }^{18}$ N. Tagg, ${ }^{23}$ W. Tang,${ }^{33}$ K. Terao,${ }^{29}$ R. T. Thornton, ${ }^{17}$ M. Toups, ${ }^{11}$ Y.-T. Tsai ${ }^{29}$ S. Tufanli, ${ }^{38}$ M. A. Uchida, ${ }^{4}$ T. Usher, ${ }^{29}$ W. Van De Pontseele, ${ }^{24,13}$ R. G. Van de Water, ${ }^{17}$ B. Viren, ${ }^{2}$ M. Weber ${ }^{1}$ H. Wei ${ }^{2}$ D. A. Wickremasinghe, ${ }^{26}$ Z. Williams,${ }^{34}$ S. Wolbers, ${ }_{11}^{11}$ T. Wongjirad ${ }^{35}$ K. Woodruff ${ }^{22}$ M. Wospakrik,,${ }^{11}$ W. Wu, ${ }^{11}$ T. Yang, ${ }^{11}$ G. Yarbrough, ${ }^{33}$ L. E. Yates, ${ }^{19}$ G. P. Zeller, ${ }^{11}$ J. Zennamo, ${ }^{11}$ and C. Zhang ${ }^{2}$

(The MicroBooNE Collaboration)*

\author{
${ }^{1}$ Universität Bern, Bern CH-3012, Switzerland \\ ${ }^{2}$ Brookhaven National Laboratory (BNL), Upton, New York 11973, USA \\ ${ }^{3}$ University of California, Santa Barbara, California 93106, USA \\ ${ }^{4}$ University of Cambridge, Cambridge CB3 OHE, United Kingdom \\ ${ }^{5}$ St. Catherine University, Saint Paul, Minnesota 55105, USA \\ ${ }^{6}$ University of Chicago, Chicago, Illinois 60637, USA \\ ${ }^{7}$ University of Cincinnati, Cincinnati, Ohio 45221, USA \\ ${ }^{8}$ Colorado State University, Fort Collins, Colorado 80523, USA \\ ${ }^{9}$ Columbia University, New York, New York 10027, USA \\ ${ }^{10}$ Davidson College, Davidson, North Carolina 28035, USA \\ ${ }^{11}$ Fermi National Accelerator Laboratory (FNAL), Batavia, Illinois 60510, USA \\ ${ }^{12}$ Universidad de Granada, E-18071 Granada, Spain \\ ${ }^{13}$ Harvard University, Cambridge, Massachusetts 02138, USA \\ ${ }^{14}$ Illinois Institute of Technology (IIT), Chicago, Illinois 60616, USA \\ ${ }^{15}$ Kansas State University (KSU), Manhattan, Kansas 66506, USA \\ ${ }^{16}$ Lancaster University, Lancaster LA1 4YW, United Kingdom \\ ${ }^{17}$ Los Alamos National Laboratory (LANL), Los Alamos, New Mexico 87545, USA \\ ${ }^{18}$ The University of Manchester, Manchester M13 9PL, United Kingdom \\ ${ }^{19}$ Massachusetts Institute of Technology (MIT), Cambridge, Massachusetts 02139, USA \\ ${ }^{20}$ University of Michigan, Ann Arbor, Michigan 48109, USA \\ ${ }^{21}$ University of Minnesota, Minneapolis, Minnesota 55455, USA \\ ${ }^{22}$ New Mexico State University (NMSU), Las Cruces, New Mexico 88003, USA \\ ${ }^{23}$ Otterbein University, Westerville, Ohio 43081, USA \\ ${ }^{24}$ University of Oxford, Oxford OX1 3RH, United Kingdom \\ ${ }^{25}$ Pacific Northwest National Laboratory (PNNL), Richland, Washington 99352, USA \\ ${ }^{26}$ University of Pittsburgh, Pittsburgh, Pennsylvania 15260, USA
}




\author{
${ }^{27}$ Rutgers University, New Brunswick, New Jersey 08901, USA \\ ${ }^{28}$ Saint Mary's University of Minnesota, Winona, Minnesota 55987, USA \\ ${ }^{29}$ SLAC National Accelerator Laboratory, Menlo Park, California 94025, USA \\ ${ }^{30}$ South Dakota School of Mines and Technology (SDSMT), Rapid City, South Dakota 57701, USA \\ ${ }^{31}$ Syracuse University, Syracuse, New York 13244, USA \\ ${ }^{32}$ Tel Aviv University, Tel Aviv 69978, Israel \\ ${ }^{33}$ University of Tennessee, Knoxville, Tennessee 37996, USA \\ ${ }^{34}$ University of Texas, Arlington, Texas 76019, USA \\ ${ }^{35}$ Tufts University, Medford, Massachusetts 02155, USA \\ ${ }^{36}$ Center for Neutrino Physics, Virginia Tech, Blacksburg, Virginia 24061, USA \\ ${ }^{37}$ University of Warwick, Coventry CV4 7AL, United Kingdom \\ ${ }^{38}$ Wright Laboratory, Department of Physics, Yale University, New Haven, Connecticut 06520, USA
}

(Received 26 November 2019; accepted 11 February 2020; published 4 March 2020)

\begin{abstract}
We present upper limits on the production of heavy neutral leptons (HNLs) decaying to $\mu \pi$ pairs using data collected with the MicroBooNE liquid-argon time projection chamber (TPC) operating at Fermilab. This search is the first of its kind performed in a liquid-argon TPC. We use data collected in 2017 and 2018 corresponding to an exposure of $2.0 \times 10^{20}$ protons on target from the Fermilab Booster Neutrino Beam, which produces mainly muon neutrinos with an average energy of $\approx 800 \mathrm{MeV}$. HNLs with higher mass are expected to have a longer time of flight to the liquid-argon TPC than Standard Model neutrinos. The data are therefore recorded with a dedicated trigger configured to detect HNL decays that occur after the neutrino spill reaches the detector. We set upper limits at the $90 \%$ confidence level on the element $\left|U_{\mu 4}\right|^{2}$ of the extended PMNS mixing matrix in the range $\left|U_{\mu 4}\right|^{2}<(6.6-0.9) \times 10^{-7}$ for Dirac HNLs and $\left|U_{\mu 4}\right|^{2}<$ $(4.7-0.7) \times 10^{-7}$ for Majorana HNLs, assuming HNL masses between 260 and $385 \mathrm{MeV}$ and $\left|U_{e 4}\right|^{2}=\left|U_{\tau 4}\right|^{2}=0$.
\end{abstract}

DOI: 10.1103/PhysRevD.101.052001

\section{INTRODUCTION}

The Standard Model (SM) describes massless neutrinos as left-handed states. The observation of neutrino oscillations [1] has demonstrated, however, that neutrinos must have mass, requiring extensions of the SM, such as the neutrino minimal Standard Model ( $\nu \mathrm{MSM})[2,3]$. The $\iota \mathrm{MSM}$ predicts additional right-handed neutral leptons that, unlike SM neutrinos, are not charged under the weak interaction and thus manifest themselves only through their mixing with SM neutrinos. According to the $\nu \mathrm{MSM}$, a right-handed neutral lepton at the $\mathrm{keV}$ mass scale can provide a candidate for dark matter, while the other two right-handed leptons are expected to have masses at the $\mathrm{GeV}$ scale [2,3]. In general, the masses of these righthanded states and their coupling to SM neutrinos are not predicted by the model, and their allowed values can thus span many orders of magnitude. In this paper, we report results from a search for heavy neutral leptons (HNLs) with masses of $\mathcal{O}(100) \mathrm{MeV}$.

\footnotetext{
"microboone_info@fnal.gov
}

Published by the American Physical Society under the terms of the Creative Commons Attribution 4.0 International license. Further distribution of this work must maintain attribution to the author(s) and the published article's title, journal citation, and DOI. Funded by SCOAP.
The MicroBooNE detector [4] began collecting data from the Booster Neutrino Beam (BNB) [5] in 2015, making it the first fully operational detector of the three liquid-argon time projection chambers comprising the Short-Baseline Neutrino Program (SBN) [6]. The SBN program will address the short-baseline anomalies observed by the MiniBooNE and LSND Collaborations $[7,8]$. One possible explanation of these anomalies is the existence of light sterile neutrinos with masses of the order of $\mathrm{eV}$, which would lead to short-baseline neutrino flavor oscillations not expected in the SM.

In addition to the studies of the effects of $\mathrm{eV}$-scale sterile neutrinos, the energy range of the BNB allows us to extend the sensitivity of the SBN detectors to the production and decay of HNLs with masses of $\mathcal{O}(100) \mathrm{MeV}$. HNLs produced by the BNB would travel along the beam line and could then decay in flight to $\mu \pi$ pairs inside the MicroBooNE detector, located $463 \mathrm{~m}$ downstream from the neutrino production target. Due to their mass, some of the HNLs are expected to arrive late compared to the arrival of the BNB spill. To suppress background from SM neutrino interactions, we therefore use data collected with a dedicated HNL trigger. This trigger was commissioned in 2017 and is used to search for late signatures occurring after the arrival of the SM neutrino beam spill. This trigger allows us to perform a search for HNLs in the mass range 
260-385 MeV using data taken in 2017 and 2018 that corresponds to $2.0 \times 10^{20}$ protons on target (POT).

\section{HEAVY NEUTRAL LEPTONS}

We define the HNL in terms of its relevant parameters: its mass $m_{N}$, and the elements of the extended PontecorvoMaki-Nakagawa-Sakata (PMNS) matrix $\left|U_{\alpha 4}\right|^{2}(\alpha=e, \mu, \tau)$. The flavor eigenstates of the left-handed neutrinos $\nu_{\alpha}$ are written as a linear combination of the SM neutrino mass eigenstates $\nu_{i}(i=1,2,3)$ and the heavy neutral lepton state, $N$, in the form

$$
\nu_{\alpha}=\sum_{i} U_{\alpha i} \nu_{i}+U_{\alpha 4} N
$$

HNLs can be produced (and decay) via SM gauge interactions, with a rate suppressed by the relevant $\left|U_{\alpha 4}\right|^{2}$ element through mixing-mediated interactions with SM gauge bosons. The decay of charged kaons and pions from the BNB can thus produce a flux of HNLs, which then propagate to the MicroBooNE detector, where they are assumed to decay to SM particles.

Figure 1 shows diagrams for the production and decay channels. In this paper, we only consider HNL decays to $\mu \pi$ final states, where the HNLs are produced through the process $\mathrm{K}^{+} \rightarrow \mu^{+} N$.

HNL states can include both Dirac and Majorana mass terms. Majorana HNLs would decay in equal numbers into $\mu^{+} \pi^{-}$and $\mu^{-} \pi^{+}$final states. Since the BNB with positive horn polarity used for this search produces predominantly neutrinos and not antineutrinos, Dirac HNLs could only decay through the process $N \rightarrow \mu^{-} \pi^{+}$.

Assuming $\left|U_{e 4}\right|^{2}=\left|U_{\tau 4}\right|^{2}=0$, the HNL production rates and the $\mu \pi$ decay width are both proportional to $\left|U_{\mu 4}\right|^{2}$ only [9], and we therefore place limits exclusively on the $\left|U_{\mu 4}\right|^{2}$ mixing matrix element. The accessible HNL masses are given by the requirement that the decay and production be kinematically allowed, i.e., $m_{K}-m_{\mu}>m_{N}>$ $m_{\mu}+m_{\pi}$.

The angular distributions of the decay products is given by the angle between the polarization vector of the HNL and the momentum of the charged lepton in the HNL rest frame. The angular distributions differ between the charge combinations $\left(\mu^{+} \pi^{-}\right.$and $\left.\mu^{-} \pi^{+}\right)$but the combined distribution describing Majorana HNLs is isotropic, and the expected rate is double the rate of Dirac HNL decays $[10,11]$.

\section{HNL FLUX IN THE BNB}

The BNB impinges protons from the Fermilab Booster synchrotron on a beryllium target. The protons are delivered in a spill with a duration of $1.6 \mu \mathrm{s}$ and an average repetition rate of up to $5 \mathrm{~Hz}$ [5]. The proton kinetic energy of $8 \mathrm{GeV}$ limits the types of mesons produced by $p$-Be

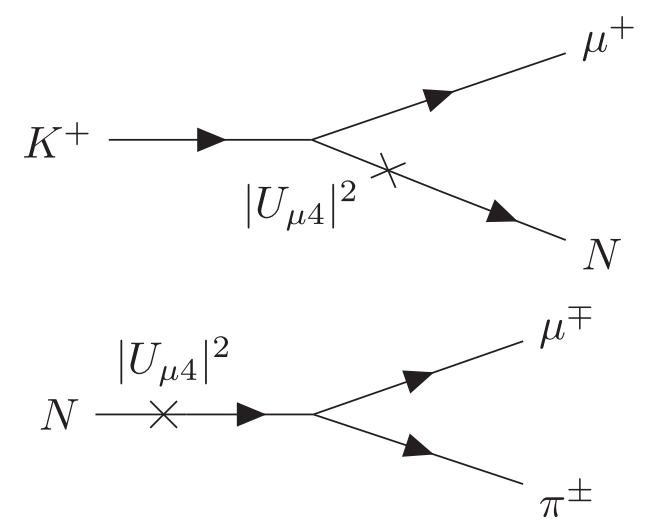

FIG. 1. Production of an HNL (labeled $N$ ) via mixing in a $K^{+}$ meson decay and its subsequent decay into a $\mu^{\mp} \pi^{ \pm}$pair.

interactions to kaons and pions, generating a muonneutrino beam with average energies of $800 \mathrm{MeV}$. This restricts the highest mass of HNL that can be produced at the BNB to $m_{K}-m_{\mu}$ for $\left|U_{\mu 4}\right|^{2}$ mediated channels.

We calculate the HNL production rate from the BNB using the SM neutrino flux simulation [5]. The decay kinematics of each SM neutrino parent are calculated for an HNL of mass $M_{N}$. Each event is then weighted by a kinematic factor to account for the effect of $M_{N}$ on the parent decay rate and by a geometric factor describing the probability of the HNL reaching the MicroBooNE detector. The geometric factor enhances the flux since the HNLs with higher mass are boosted into the beam direction.

The kinematic factor suppresses HNL production at the kinematic threshold and takes into account the smaller helicity suppression due to the mass of the HNLs [12-14].

Due to the Lorentz transformation into the lab frame, the decay probability becomes inversely proportional to HNL momentum. In contrast, the number of SM neutrino interactions is given by their interaction cross sections on argon, which rises with energy. The HNL flux is thus expected to be enhanced at lower momenta, leading to correspondingly longer travel times to the detector.

\section{MicroBooNE DETECTOR}

The MicroBooNE detector [4] is a liquid-argon time projection chamber (TPC) situated at near-ground level at a location $463 \mathrm{~m}$ downstream from the target of BNB, receiving a $93.6 \%$ pure $\nu_{\mu}$ beam. The MicroBooNE TPC has an active mass of $85 \mathrm{t}$ of liquid argon, in a volume $2.6 \times 2.3 \times 10.4 \mathrm{~m}^{3}$ in the $x, y, z$ coordinates, respectively. The MicroBooNE detector is described by a right-handed coordinate system. The $x$ axis points along the negative drift direction with the origin located at the anode plane, the $y$ axis points vertically upward with the origin at the center of the detector, and the $z$ axis points along the direction of the beam, with the origin at the upstream edge of the detector. The polar angle is defined with 


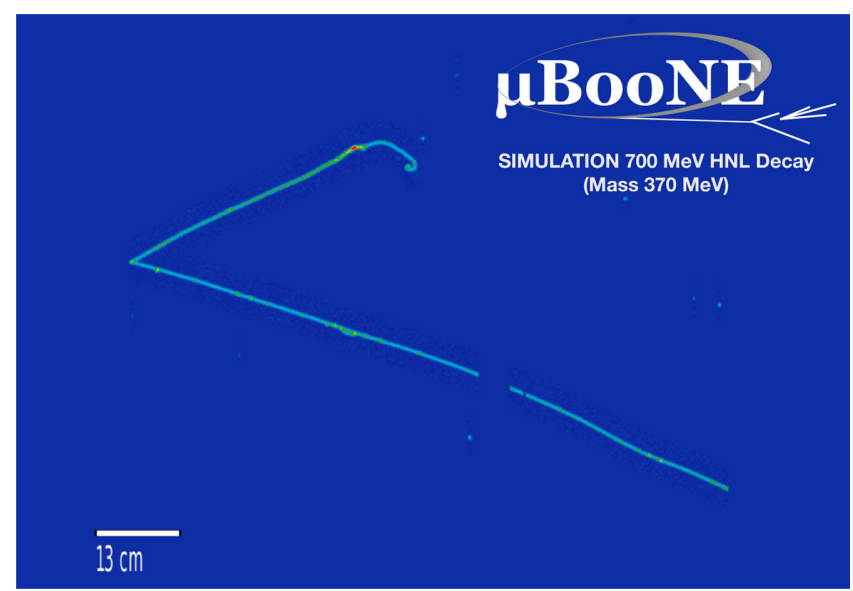

FIG. 2. Display of a $\mu \pi$ decay for an HNL with a mass of $370 \mathrm{MeV}$, showing the signals measured at the collection plane. The horizontal axis represents the wire number, and the vertical axis represents time. Colors show the charge deposition measured on the wires. The gap in the longer track is due to a set of unresponsive wires.

respect to the $z$ axis and the azimuthal angle $\phi$ with respect to the $y$ axis.

Neutrinos that cross the detector can interact with the argon nuclei, or, in the case of HNLs, decay to SM particles and produce secondary charged particles that ionize the argon atoms along their trajectories producing ionization electrons and scintillation light. An electric field of $273 \mathrm{~V} / \mathrm{cm}$ causes the electrons to drift towards the anode plane, requiring $2.3 \mathrm{~ms}$ to drift across the width of the detector. The anode planes are positioned perpendicular to the electric field and comprise three planes of sense wires with a spacing of $3 \mathrm{~mm}$ between adjacent wires and the same spacing separating the wire planes. Ionization electrons induce a bipolar signal when they pass through the first two planes of wires, oriented at $\pm 60^{\circ}$ with respect to the vertical, before being collected on the third plane with vertically oriented wires producing a unipolar signal.

The waveforms measured by the 8192 wires are digitized in a $4.8 \mathrm{~ms}$ readout window. The signal processing on the raw TPC waveforms includes noise filtering and deconvolution to convert wire signals into hit information [15]. Subsequently, individual hits corresponding to a localized energy deposit are extracted for each wire. The combination of timing information and energy deposit contained in each waveform is used to create two-dimensional (2D) projective views of the event. Figure 2 shows such a 2D view for a simulated HNL decay. The Pandora [16,17] toolkit is then used to reconstruct three-dimensional (3D) tracks (produced by muons, pions and protons) and showers (produced by electrons and photons) from the $2 \mathrm{D}$ views.

A calibration is performed to take into account all the microphysics in the detector, including electron-ion recombination and the space charge effect (SCE) [18]. The SCE is caused by slowly drifting ions produced by cosmic rays that create variations in the electric fields. This variation impacts energy deposits and track trajectories, which appear distorted, particularly near the edges of the TPC. The individual energy deposits are corrected by the timeaveraged calibration factors obtained from data.

An array of 32 8-inch photomultiplier tubes (PMTs) with $16 \mathrm{~ns}$ timing resolution collects the scintillation light produced by argon ionization. We use this measurement to determine the time of the neutrino interaction and for triggering. Light flashes are reconstructed with a timing resolution of $100 \mathrm{~ns}$ by summing waveforms from the 32 PMTs.

\section{TRIGGERS AND DATA SAMPLES}

To reduce the amount of recorded data, online software triggers are deployed, processing the waveforms of the light collection system so that activities in coincidence with BNB spills are identified and stored. SM neutrinos arrive at the MicroBooNE detector $1.5 \mu$ s after they have been produced, while the time of flight of the HNLs depends on their mass and momentum. As the HNL mass increases, an increasing fraction of HNL events would thus arrive at the detector after the end of the BNB trigger window of $1.9 \mu \mathrm{s}$.

HNLs decaying into $\mu \pi$ pairs within the BNB trigger window need to be discriminated from HNL-like backgrounds due to SM charged-current neutrino interactions that include muons, pions, and also protons in the final state. Neutrino-induced charged-current coherent pion production, where no additional activity around the vertex is expected, could present a nearly irreducible background. The only background relevant for HNL decays that occur after the end of the BNB trigger window are crossing cosmic-ray muons, which have a distinctly different topology from the signal. We therefore focus on the HNLs arriving after the end of the BNB trigger window in this analysis.

In June 2017, we introduced an HNL trigger that starts concurrently with the BNB trigger-in coincidence with the beam spill arrival-but extends the trigger window from 1.9 to $2.5 \mu$ s. Figure 3 shows the relationship between the time of flight, the initial energy of HNLs, and the trigger windows, illustrating that the HNL trigger retains a larger fraction of HNL decays when the HNL is heavier. The HNL trigger requires the number of photoelectrons $\left(N_{p e}\right)$ recorded by the PMTs to be $N_{p e}>10.5$, which is slightly higher than the requirement of $N_{p e}>6.5$ for the BNB trigger. This choice optimizes signal efficiency and trigger rate.

The MicroBooNE detector is exposed to a large flux of cosmic-ray muons, traversing at a rate of $\approx 5.5 \mathrm{kHz}$, since it is situated just below ground level at a depth of $\approx 6 \mathrm{~m}$ with no significant overburden. Events in coincidence with BNB 


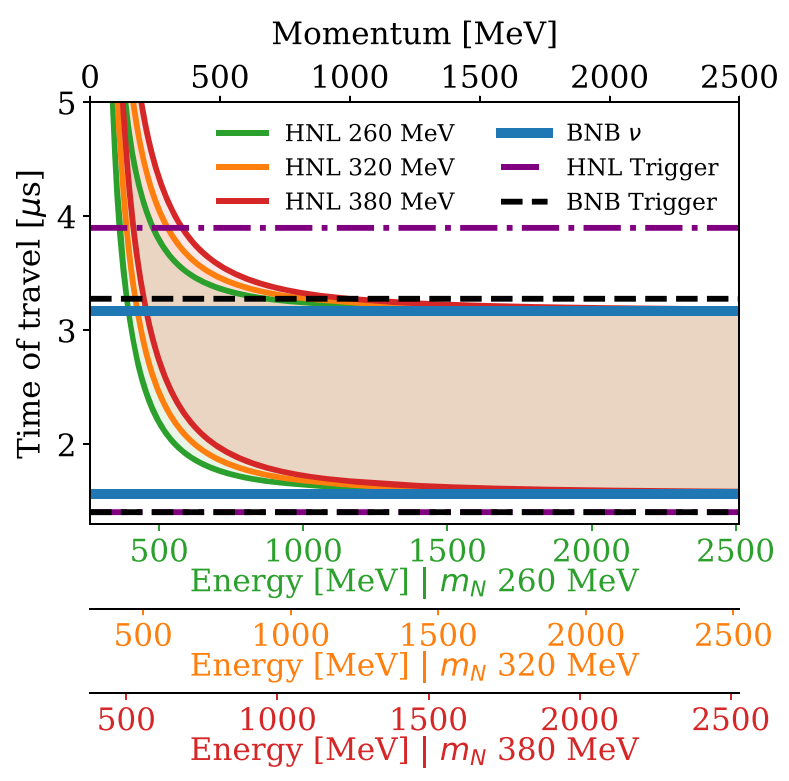

FIG. 3. Time of travel from the BNB target to the MicroBooNE detector for SM active neutrinos and HNLs of different masses. Blue solid lines indicate time of travel for SM active neutrinos, produced within a beam spill of $1.6 \mu \mathrm{s}$. Neutrinos are expected to arrive at any time between the two solid lines independent of their initial momentum. The dashed lines indicate the start (black at $1.4 \mu \mathrm{s}$ ) and end time of the BNB (black) and HNL (purple) trigger. The solid lines and bands indicate the time of travel for HNLs of different mass within a spill.

spill ("on-beam") data contain up to $\approx 20$ cosmic-ray muons within the readout window of $4.8 \mathrm{~ms}$.

For background studies, we collect data sets based on identical trigger settings as for on-beam data, with the exception of the beam coincidence requirement. These "off-beam" data sets were taken with either the BNB or the HNL trigger requirement on the number of photoelectrons. They contain mainly cosmic rays and no SM neutrino interactions.

In summary, we make use of three data samples:

(1) On-beam HNL data, taken in coincidence with a BNB neutrino spill. This data set requires an event to fulfil the HNL trigger condition with a veto on the $\mathrm{BNB}$ trigger to reject activity produced by neutrino interactions during the BNB spill.

(2) On-beam BNB data, taken in coincidence with a BNB neutrino spill and fulfilling the BNB trigger conditions.

(3) Off-beam data, taken with identical trigger settings and in a time window of the same length as for the on-beam BNB or HNL data, but at a time when no beam spills are received at the detector.

\section{MONTE CARLO SAMPLES}

Simulated data sets are used to evaluate the reconstruction and selection efficiency, to train a boosted

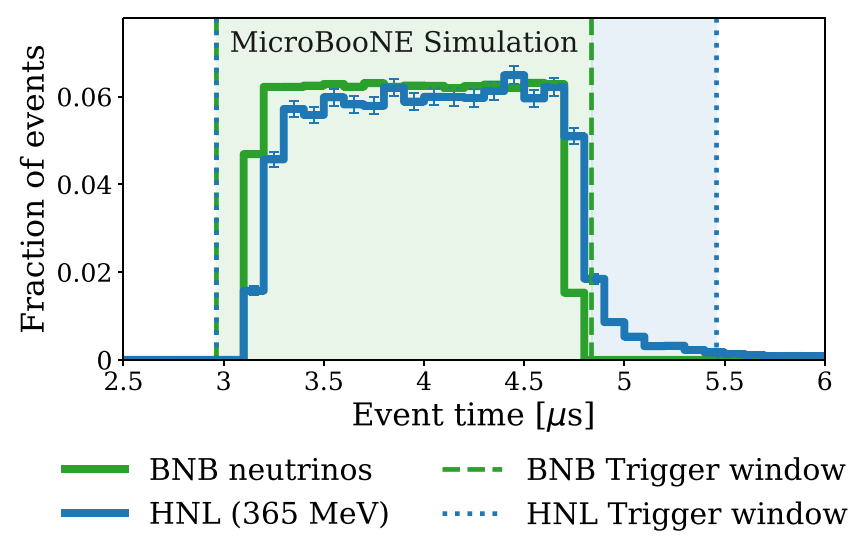

FIG. 4. Timing distribution for muon neutrinos and HNLs produced in the BNB. The HNL mass is $365 \mathrm{MeV}$. Vertical lines indicate the start and end time of the BNB and HNL trigger windows.

decision tree for signal discrimination and to provide a control sample of SM neutrino interactions for validation.

HNL signal samples are simulated for ten different HNL masses. All HNLs are assumed to travel collinearly with the beam axis, i.e., parallel to the longitudinal $z$ axis of the MicroBooNE detector, such that $p_{x}=p_{y}=0$. The time distribution of the HNL production is assumed to be uniform within the beam spill, neglecting the $\approx 19$ ns wide bunch structure, which cannot be resolved with the light reconstruction used in this analysis. The calculated arrival time distributions for HNLs and SM neutrinos produced in a BNB spill is shown in Fig. 4. We simulate HNL decays into $\mu^{-} \pi^{+}$and $\mu^{+} \pi^{-}$final states with isotropic angular distributions. The $\mu^{-} \pi^{+}$decays are reweighted to obtain the angular distribution for Dirac HNLs.

Interactions of SM neutrinos in liquid argon, as well as in the material surrounding the detector ("dirt" events), are simulated within the LArSoft [19] framework using the GENIE [20] Monte Carlo program. For HNL signal events, cosmic rays crossing the detector are modeled by overlaying data from zero-bias off-beam data events, whereas the CORSIKA [21] program is used to simulate cosmic rays for SM neutrino interactions. The detector simulation and propagation of secondary particles in liquid argon is simulated with GEANT4 [22,23].

\section{EVENT SELECTION}

As this analysis focuses on the HNLs arriving after the end of the BNB trigger window, events containing signal candidates need to pass the HNL trigger condition but not the BNB trigger condition. Since signal candidates are expected to comprise two reconstructed tracks sharing a common vertex, we select vertices reconstructed by Pandora with exactly two associated tracks. At this stage, events can contain more than one such HNL candidate. To reduce background, we apply further selections: 
(1) Fiducial Volume. The reconstructed vertex associated with the HNL candidate is required to be located in a fiducial volume, defined as a cuboid contained in the active TPC volume. The vertex location must be greater than $12 \mathrm{~cm}$ from the border of the active volume along the $x$ axis, $35 \mathrm{~cm}$ along the $y$ axis, $25 \mathrm{~cm}$ from the upstream edge of the $z$ axis, and $85 \mathrm{~cm}$ from the downstream edge. HNL candidates with vertices located within a $1 \mathrm{~m}$ wide gap along the $z$ axis in the range $675<z<775 \mathrm{~cm}$ are also rejected since the wires are not optimally performant in this region [15]. The fiducial volume thus defined corresponds to a mass of $43 \mathrm{t}$ of liquid argon.

(2) Vertex Track Distance. We require the starting points of the two tracks associated with the HNL candidate to lie within $5 \mathrm{~cm}$ from the location of the reconstructed vertex.

(3) Minimum Number of Hits. The tracks associated with an HNL candidate are required to each have more than 30 associated hits in the collection plane, which corresponds to a minimum energy deposit of $\approx 20 \mathrm{MeV}$.

(4) Flash Distance. We require the distance in the $y z$ plane between the center of the reconstructed light flash and the $y z$ projection of the vertex location to be less than $150 \mathrm{~cm}$.

(5) Track Containment. The tracks associated with an HNL candidate have to be fully contained within a volume defined by a distance of $25 \mathrm{~cm}$ from the edges of the active volume on the $y$ axis and $10 \mathrm{~cm}$ on the $x$ and $z$ axes. This requirement removes tracks crossing the TPC edges that are more severely affected by the SCE. It also rejects cosmic-ray background.

(6) Kinematics. A large fraction of cosmic-ray muons misidentified as HNL candidates are caused by "broken" tracks where the reconstruction algorithm has split the muon track, assigning the two sections to a common vertex at the point where the track is broken. Such candidates have a large opening angle $\Delta \alpha$ between the two tracks and are thus rejected by requiring $\Delta \alpha<2.8$. We also require that the mass as determined from the momenta assigned to the tracks is $<500 \mathrm{MeV}$.

The momenta of the two tracks representing the $\mu \pi$ pair from the HNL decay are determined by the length of the track under the hypothesis that the longer particle track is from the muon and the shorter the pion. The length of the tracks associated with the HNL candidates is of order $10 \mathrm{~cm}$ and depends on the HNL mass. The impact of the muonpion assignment on the results is negligible.

After applying these selection requirements, (45-50)\% of the HNL candidates in the HNL simulation are retained, with a corresponding background selection efficiency of
TABLE I. Number of candidates remaining after the selection requirements for the HNL signal with a mass of $370 \mathrm{MeV}$ and for $\left|U_{\mu 4}\right|^{2}=1.4 \times 10^{-7}$, the expected background rate derived from the off-beam data set, and the on-beam HNL data, corresponding to $2.0 \times 10^{20}$ POT. The uncertainty on the expected background rate is given by the statistical uncertainty of the off-beam data.

\begin{tabular}{lccc}
\hline \hline & HNL signal & Background & Data \\
\hline Two-track vertex & 100 & 41426 & 41914 \\
Fiducial volume & 61 & 21501 & 21811 \\
Vertex-track distance & 57 & 16126 & 16339 \\
Minimum number of hits & 57 & 15924 & 16126 \\
Flash requirement & 57 & 7487 & 7527 \\
Track containment & 47 & 1096 & 1138 \\
Kinematics (mass, angle) & 45 & $653 \pm 286$ & 669 \\
\hline \hline
\end{tabular}

1.6\%. The contribution from dirt events in the background sample are found to be negligible. Table I shows the numbers of HNL candidates for the data samples used for this analysis, corresponding to $2.0 \times 10^{20} \mathrm{POT}$, and for the corresponding HNL signal simulation assuming a mass of $370 \mathrm{MeV}$ and a mixing angle of $\left|U_{\mu 4}\right|^{2}=1.4 \times 10^{-7}$. The expected number of background candidates is derived from the off-beam data by normalizing the number of time windows in the off-beam data to the number of beam spills of the on-beam data. The background expectation agrees with the data within the statistical uncertainties.

\section{SIGNAL EXTRACTION}

We train a boosted decision tree (BDT) on a set of kinematic variables to discriminate between signal candidates and background using the XGBoost framework [24]. The following variables are used as input to the BDT:

(1) the 3D opening angle $\Delta \alpha$ between the two tracks associated with the HNL decay;

(2) the momentum $\left|p_{N}\right|$ of the HNL candidate;

(3) the polar angle $\theta$ of the HNL candidate;

(4) the azimuthal angle $\phi$ of the HNL candidate; and

(5) the invariant mass $m_{N}$ of the $\mu \pi$ pair,

where $\theta$ and $\phi$ are defined in the MicroBooNE coordinate system.

Figure 5 shows the distributions of these variables for signal HNL candidates with a mass of $370 \mathrm{MeV}$, and for the off-beam data set with the selection applied. Since we observe no statistically significant difference in the kinematic distributions between the BNB and HNL triggered off-beam data sets, the higher statistics off-beam BNB data set is shown here.

The distributions show good separation between signal and background. Background is constituted mostly of "broken" cosmic-ray tracks. We train separate BDT models for ten different mass hypotheses in the range 260-385 MeV. The BDT score distributions for the signal and for the off-beam background shown in Fig. 6 

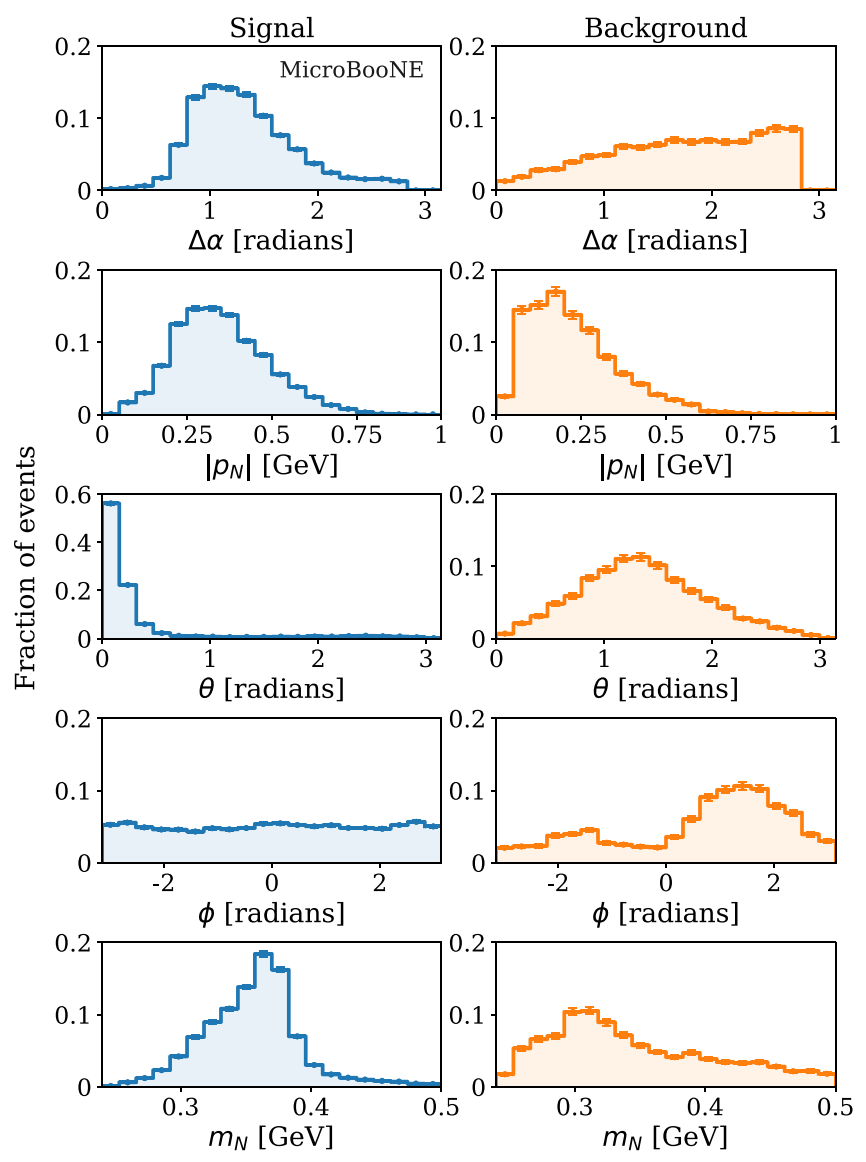

HNL Signal (370 MeV)

Off-beam data

FIG. 5. Kinematic variables used to train the BDT for the HNL candidates. The left column shows the distributions for HNL candidates with a mass of $370 \mathrm{MeV}$, and the right column shows the distributions for the off-beam data. The distributions are normalized to 1 .

demonstrate good separation between signal and background for different HNL masses. The discrimination between signal and background improves with increased HNL mass.
The distributions in Fig. 5 are given for HNL candidates, where each event can contain more than one HNL candidate. Since the probability to observe more than one HNL candidate is negligible for the mixing angles considered here, we retain only the candidate with the most signal-like BDT score in each event.

\section{CONTROL SAMPLES}

We use a statistically independent control sample to validate the determination of the reconstruction and selection efficiencies, and the BDT performance. The control sample is the on-beam BNB data set, which is expected to contain SM neutrino interactions with similar final-state topologies as HNL decays. In addition to applying the HNL selection to the control sample, we reject HNL candidates where one of the tracks has an associated energy deposit consistent with the specific energy loss expected for a proton. This gives a better representation of the HNL selection, since candidates with protons in the final state are not expected in the signal sample.

The trigger used to record the on-beam BNB sample selects events with any kind of interaction, i.e., the data sample will contain events with cosmic-rays only and no SM neutrino interaction. We therefore subtract the distributions obtained from an off-beam data sample containing cosmic-ray events to obtain distributions statistically representing a sample of mainly SM neutrino interactions.

The numbers of HNL candidates remaining after each preselection step are given in Table II for the control sample and the Monte Carlo (MC) simulation of BNB SM neutrino events. We then apply the same BDTs trained for three HNL masses as used for the signal selection in the previous section to the control samples after the selection to obtain the BDT score distributions in Fig. 7. The distributions for the MC simulation of BNB SM neutrino events are normalized to the same number of POTs as the data. We observe good agreement between data and simulation in terms of shape and normalization. For BDTs trained with
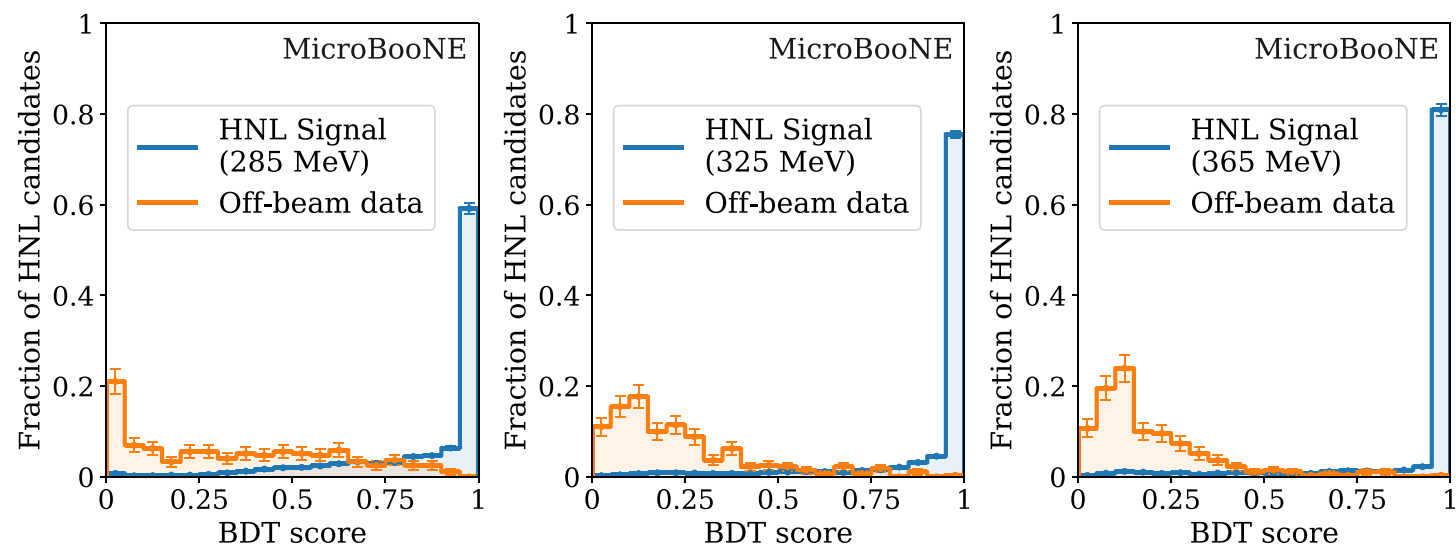

FIG. 6. BDT score distribution for three distinct BDTs trained with HNL masses of 285, 325, and $365 \mathrm{MeV}$, respectively, comparing simulated HNL signal and off-beam background data. The uncertainty on the samples is statistical. 
TABLE II. Numbers of HNL candidates remaining after the application of selection requirements to HNL candidates in the control samples, comparing simulation and data. The off-beam cosmic-ray data has been rescaled and subtracted from the onbeam data to obtain the control sample. The uncertainty on the simulation is given by the statistical uncertainty on the generated number of MC events.

\begin{tabular}{lcc}
\hline \hline & Control sample & Simulation \\
\hline Two-track vertex & 81112 & 79365 \\
Fiducial volume & 43078 & 42414 \\
Vertex-track distance & 32120 & 32471 \\
Minimum number of hits & 31939 & 32228 \\
Flash requirement & 23089 & 19962 \\
Track containment & 6344 & 6021 \\
Kinematics (mass, angle) & 3972 & $4267 \pm 475$ \\
\hline \hline
\end{tabular}

higher HNL masses, signal-like events appear at higher BDT scores. In the MC simulation, these events are mostly due to charged-current (CC) neutrino interactions. Kinematics and track length in CC neutrino interactions become more similar to the simulated kinematics for higher mass HNLs. This background is not present in the data samples used for the HNL search.

\section{SYSTEMATIC UNCERTAINTIES}

Systematic uncertainties on the simulation of the HNL signal originate from the simulation of the HNL flux, the trigger efficiency, and several calibration and reconstruction effects. To quantify the systematic uncertainties, we use a signal-enriched sample with a BDT score $>0.95$. The following uncertainties on the signal are considered:

(1) HNL flux uncertainties are estimated by simultaneously varying all parameters used for the flux simulation [25]. The relevant parameters are related to the beam-line simulation, which includes variations of the horn current and the skin effect, and to uncertainties on the kaon production cross section. The contributions to the total flux uncertainty from both types of uncertainty are approximately equal. For all HNL masses, the overall uncertainty on the flux is $8 \%$. In addition, a constant $2 \%$ uncertainty accounts for the POT counting performed with the beam toroid.

(2) Systematic uncertainties on the trigger efficiency originate from the timing resolution of the PMTs, which we use to define the HNL trigger window and vetoes of the BNB trigger window. Uncertainties caused by variations of the light yield are negligible. The trigger uncertainty is estimated to be in the range (5-10)\% depending on the mass of the HNL signal.

(3) Dynamically induced charge (DIC) refers to the charge induced on the wires beyond the wire closest to the ionization trail $[26,27]$. This effect impacts the algorithm that determines the deposited charge, especially on induction planes, as well as the pattern recognition. To estimate the impact of DIC effects, we compare samples where DIC is simulated in the region up to the adjacent 20 wires (10 on each side) with a MC simulation that does not model DIC. The resulting uncertainty on the normalization of the signal sample is $\approx 10 \%$.

(4) Distortions caused by the SCE are corrected by timeaveraged calibration factors obtained from data. We estimate the uncertainties originating from the SCE correction by comparing the reconstruction efficiency in the MC samples with and without the simulation of the SCE. The corresponding uncertainty is found to be negligible $(<1 \%)$.

(5) The uncertainties from the remaining detector effects (such as recombination, attenuation and diffusion) are determined by comparing HNL MC samples that
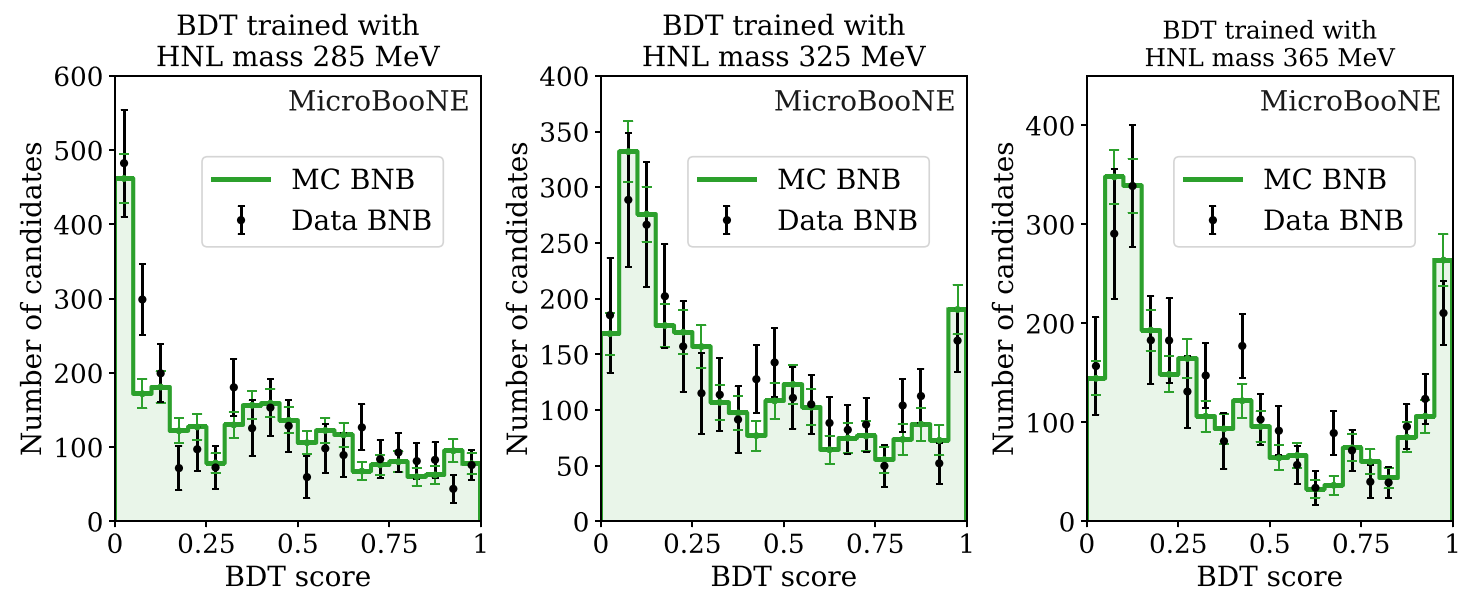

FIG. 7. BDT score distributions for three distinct BDTs trained with HNL masses of 285, 325, and $365 \mathrm{MeV}$, respectively, of the MC $\mathrm{BNB}$, and the on-beam BNB data subtracting the off-beam BNB control data samples. The uncertainty on the samples is statistical. Signal-like events are expected at higher BDT scores. 
TABLE III. Systematic uncertainties in the signal sample with BDT score $>0.95$ for an HNL mass value of $325 \mathrm{MeV}$.

\begin{tabular}{lc}
\hline \hline Source & Uncertainty \\
\hline HNL flux & $8 \%$ \\
POT & $2 \%$ \\
Trigger & $8 \%$ \\
Dynamically induced charge & $10 \%$ \\
Space charge effect & $0.3 \%$ \\
Detector response & $0.4 \%$ \\
Total & $15 \%$ \\
\hline \hline
\end{tabular}

only differ in the simulation of the detector response. The response is either obtained from data after detector calibration or from simulation. The resulting uncertainties due to the detector response are estimated to be small $(<1 \%)$.

Uncertainties from nuclear interaction modeling are negligible, since the main background source is cosmic-ray muons. For Dirac HNLs, we consider only $\mu^{-} \pi^{+}$decays and not the sum of the charge combinations. The difference in efficiency between the charge combinations is $\approx(2-3) \%$, which is small compared to the total systematic uncertainty. This difference is thus neglected.

The contribution of the systematic uncertainties on the signal efficiency in the signal-enriched sample are summarized in Table III for a HNL mass value of $325 \mathrm{MeV}$. The systematic uncertainties grow linearly with HNL mass, from $10 \%$ to $18 \%$ in the mass range $260-385 \mathrm{MeV}$. Uncertainties on the background estimation, which is derived from data, is dominated by the statistical fluctuations of the off-beam HNL data sample.

\section{RESULTS AND DISCUSSION}

The BDT score distributions for signal, background expectation (off-beam HNL data) and data are shown in
Fig. 8. Signal and background are well separated, and no data excess is observed in the signal region with high BDT scores. We therefore proceed to set limits on the HNL production rate as a function of mass.

The limits are determined using the modified frequentist $\mathrm{CL}_{s}$ method [28-30]. We calculate a log-likelihood ratio (LLR) test statistic using Poisson probabilities for estimated background events, signal yields, and the observed number of events for different HNL mass hypotheses. The confidence levels are derived by integrating the LLR distribution in pseudoexperiments using both the signalplus-background $\left(\mathrm{CL}_{s+b}\right)$ and the background-only hypotheses $\left(\mathrm{CL}_{b}\right)$. The excluded signal rate is defined by the signal strength for which the confidence level for signal, $\mathrm{CL}_{s}=\mathrm{CL}_{s+b} / \mathrm{CL}_{b}$, equals 0.1 .

Systematic uncertainties on both background and signal are taken into account using Gaussian priors. The total systematic uncertainty of approximately $15 \%$ on the signal is found to have a negligible impact on the sensitivity, since the uncertainty is dominated by the statistics of the background sample.

To calculate limits, we split the distribution into a signalenriched and signal-depleted region with BDT scores $>0.95$ and $0.5<$ BDT score $<0.95$, respectively. In Table IV, we compare the expected number of background events to the data for the different HNL mass hypotheses, as well as for the signal-enriched and signal-depleted samples. The expected number of HNL signal events is calculated assuming $\left|U_{\mu 4}\right|^{2}=1.4 \times 10^{-7}$.

The observed upper limits at the $90 \%$ confidence level as a function of mass are presented in Fig. 9 and Table V for Majorana HNLs, together with the median expected limit and the 1 and 2 standard deviation bands on the median expected limit. The bands are asymmetric since Poisson statistics are used where the expected number of events is small. The observed and expected limit agree within 1 standard deviation over the entire mass range. The decay
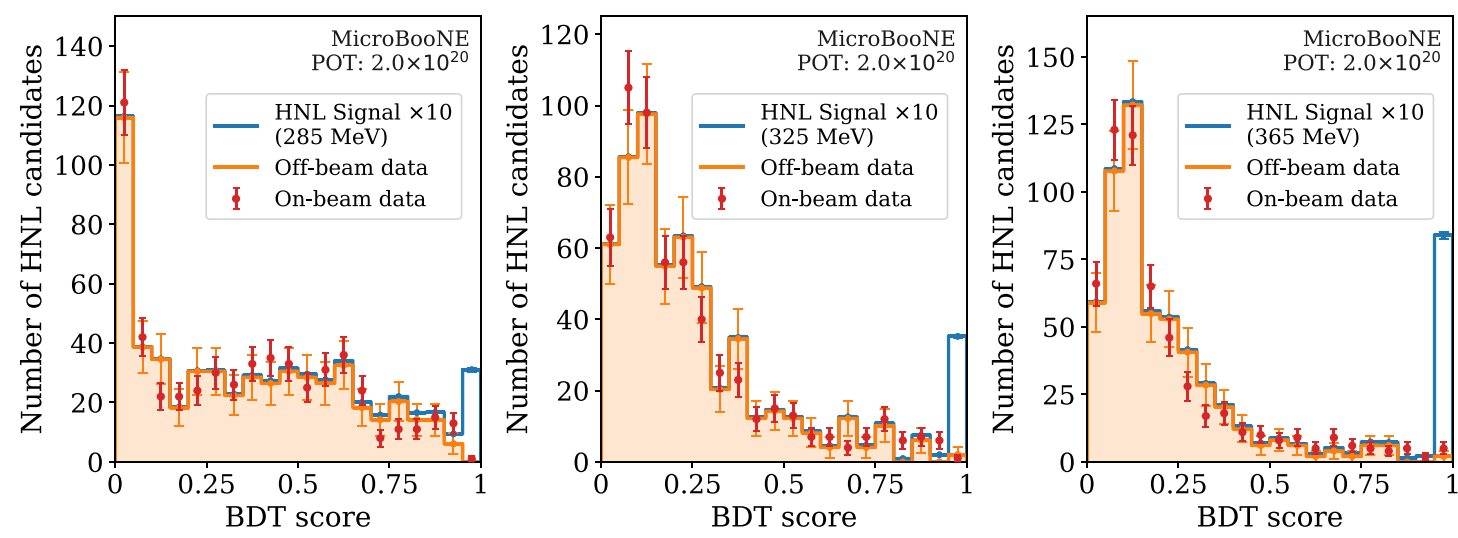

FIG. 8. BDT score distribution for the on-beam HNL and background off-beam HNL data samples. The signal distribution is shown as a stacked histogram added to the background (off-beam data), with the normalization fixed at the $90 \%$ C.L., multiplied by a factor of 10 . The statistical uncertainties for the data samples are shown separately. 
TABLE IV. Number of events with a BDT score $>0.95$ and a BDT score in the range 0.5 to 0.95 for an HNL signal with $\left|U_{\mu 4}\right|^{2}=1.4 \times 10^{-7}$, for the expected background, and for the on-beam HNL data. Systematic uncertainties are given for the signal and statistical uncertainties for the background. The $68 \%$ C.L. Poisson interval is used for bins with zero expected background events.

\begin{tabular}{lccccccc}
\hline \hline \multirow{2}{*}{$\begin{array}{l}\text { Mass } \\
(\mathrm{MeV})\end{array}$} & \multicolumn{2}{c}{ BDT score $>0.95$} & & \multicolumn{2}{c}{ BDT score $0.5-0.95$} \\
\cline { 2 - 3 } \cline { 7 - 8 } \cline { 6 - 8 } & HNL & Bkg. & Data & & HNL & Bkg. & Data \\
\hline 260 & $0.21 \pm 0.03$ & $<3.7$ & 1 & & $0.43 \pm 0.06$ & $169 \pm 19$ & 170 \\
265 & $0.42 \pm 0.06$ & $2 \pm 2$ & 1 & & $0.6 \pm 0.1$ & $185 \pm 19$ & 205 \\
285 & $1.6 \pm 0.3$ & $<3.7$ & 3 & & $0.8 \pm 0.1$ & $175 \pm 19$ & 174 \\
300 & $2 \pm 0.3$ & $2 \pm 2$ & 1 & & $1.0 \pm 0.2$ & $126 \pm 16$ & 121 \\
305 & $4 \pm 0.6$ & $2 \pm 2$ & 4 & & $0.8 \pm 0.1$ & $61 \pm 11$ & 80 \\
325 & $6 \pm 1$ & $2 \pm 2$ & 0 & & $1.6 \pm 0.3$ & $57 \pm 11$ & 69 \\
345 & $12 \pm 2$ & $2 \pm 2$ & 4 & & $2 \pm 0.3$ & $59 \pm 11$ & 69 \\
365 & $20 \pm 3$ & $2 \pm 2$ & 5 & & $2 \pm 0.3$ & $35 \pm 8$ & 53 \\
370 & $24 \pm 4$ & $2 \pm 2$ & 4 & & $4 \pm 0.6$ & $37 \pm 9$ & 47 \\
385 & $36 \pm 6$ & $<3.7$ & 4 & & $4 \pm 0.6$ & $20 \pm 6$ & 28 \\
\hline \hline
\end{tabular}

rates for Dirac HNLs are a factor of 2 smaller, and we observe no significant difference between the efficiencies to observe Majorana and Dirac HNLs. Limits for the Dirac case are therefore derived by multiplying the values in Table $\mathrm{V}$ by a factor of $\sqrt{2}$.

The results are of similar or better sensitivity as those obtained by the NA62 [31] and NuTeV [32] Collaborations for the same mixing parameter in the overlapping $\mathrm{HNL}$ mass range. The E949 Collaboration [33] sets limits in a lower mass range between 175 and $300 \mathrm{MeV}$ by measuring $\mathrm{K}^{+}$meson decays at rest. The PS191 [34,35] and T2K [36] Collaborations place more stringent limits in the HNL mass range between 260 and $360 \mathrm{MeV}$, but their location at an off-axis angle of 2 degrees restricts the sensitivity to slightly lower masses. The MicroBooNE detector is located

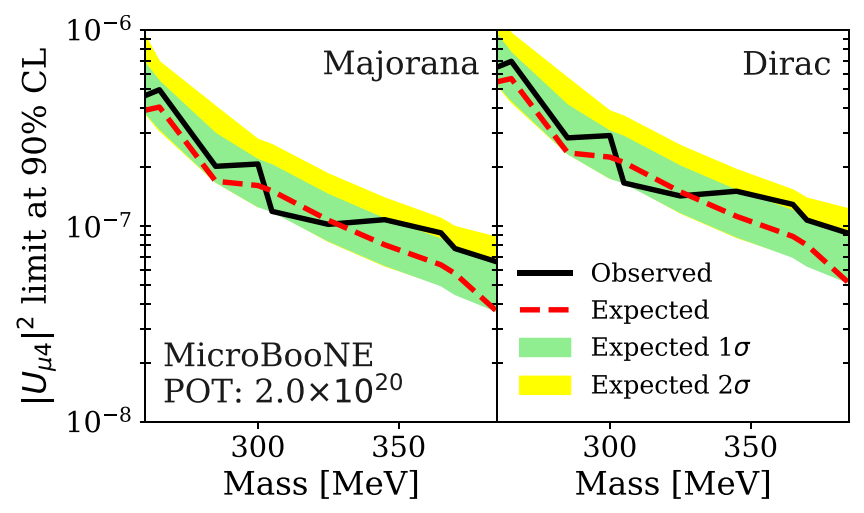

FIG. 9. Limits on $\left|U_{\mu 4}\right|^{2}$ at the $90 \%$ confidence level as function of mass for a Majorana and Dirac HNL decaying into $\mu \pi$ pairs. The observed limit is compared to the median expected limit with the 1 and 2 standard deviation $(\sigma)$ bands.
TABLE V. Limits on $\left|U_{\mu 4}\right|^{2}$ at the $90 \%$ confidence level for Majorana HNLs decaying into $\mu \pi$ pairs, multiplied by a factor of $10^{7}$. The Majorana HNL limits are multiplied by a factor of $\sqrt{2}$ to obtain the Dirac HNL limits.

\begin{tabular}{lccc}
\hline \hline Mass $(\mathrm{MeV})$ & Observed & Median Expected & $1 \sigma$ band \\
\hline 260 & 4.65 & 3.92 & $3.81-6.78$ \\
265 & 4.98 & 4.06 & $3.16-5.49$ \\
285 & 2.03 & 1.70 & $1.69-2.97$ \\
300 & 2.08 & 1.62 & $1.27-2.19$ \\
305 & 1.19 & 1.52 & $1.20-2.05$ \\
325 & 1.02 & 1.08 & $0.84-1.41$ \\
345 & 1.08 & 0.80 & $0.63-1.05$ \\
365 & 0.92 & 0.63 & $0.50-0.86$ \\
370 & 0.77 & 0.57 & $0.45-0.77$ \\
385 & 0.65 & 0.36 & $0.36-0.63$ \\
\hline \hline
\end{tabular}

on axis, allowing it to set the most constraining limits in the mass range up to $385 \mathrm{MeV}$.

\section{CONCLUSION}

We present the first search for HNLs in a liquid-argon TPC using data recorded with the MicroBooNE detector in 2017-2018 with a novel trigger recording events that arrive at the MicroBooNE detector after the BNB beam spill. The data correspond to $2.0 \times 10^{20}$ POT. We assume that the HNLs are produced in kaon decays and decay exclusively into a muon pion final state. We obtain constraints on the element $\left|U_{\mu 4}\right|^{2}$ of the extended PMNS mixing matrix of $\left|U_{\mu 4}\right|^{2}<(4.7-0.7) \times 10^{-7}$ for Majorana HNLs and $\left|U_{\mu 4}\right|^{2}<(6.6-0.9) \times 10^{-7}$ for Dirac HNLs with masses between 260 and $385 \mathrm{MeV}$ and assuming $\left|U_{e 4}\right|^{2}=\left|U_{\tau 4}\right|^{2}=0$.

\section{ACKNOWLEDGMENTS}

This document was prepared by the MicroBooNE Collaboration using the resources of the Fermi National Accelerator Laboratory (Fermilab), a U.S. Department of Energy, Office of Science, HEP User Facility. Fermilab is managed by Fermi Research Alliance, LLC (FRA), acting under Contract No. DE-AC02-07CH11359. MicroBooNE is supported by the following: the U.S. Department of Energy, Office of Science, Offices of High Energy Physics and Nuclear Physics; the U.S. National Science Foundation; the Swiss National Science Foundation; the Science and Technology Facilities Council (STFC), part of the United Kingdom Research and Innovation; and The Royal Society (United Kingdom). Additional support for the laser calibration system and cosmic ray tagger was provided by the Albert Einstein Center for Fundamental Physics, Bern, Switzerland. 
[1] M. Tanabashi et al. (Particle Data Group), Review of particle physics, Phys. Rev. D 98, 030001 (2018), and references therein.

[2] T. Asaka and M. Shaposhnikov, The $\nu$ MSM, dark matter and baryon asymmetry of the universe, Phys. Lett. B 620, 17 (2005).

[3] T. Asaka, S. Blanchet, and M. Shaposhnikov, The $\nu$ MSM, dark matter and neutrino masses, Phys. Lett. B 631, 151 (2005).

[4] R. Acciarri et al. (MicroBooNE Collaboration), Design and construction of the MicroBooNE detector, J. Instrum. 12, P02017 (2016).

[5] A. A. Aguilar-Arevalo et al. (MiniBooNE Collaboration), The neutrino flux prediction at MiniBooNE, Phys. Rev. D 79, 072002 (2009).

[6] P. A. Machado, O. Palamara, and D. W. Schmitz, The shortbaseline neutrino program at Fermilab, Annu. Rev. Nucl. Part. Sci. 69 (2019).

[7] A. Aguilar-Arevalo et al. (LSND Collaboration), Evidence for neutrino oscillations from the observation of $\bar{\nu}_{e}$ appearance in a $\bar{\nu}_{\mu}$ beam, Phys. Rev. D 64, 112007 (2001).

[8] A. A. Aguilar-Arevalo et al. (MiniBooNE Collaboration), Significant Excess of Electron-Like Events in the MiniBooNE Short-Baseline Neutrino Experiment, Phys. Rev. Lett. 121, 221801 (2018).

[9] A. Atre, T. Han, S. Pascoli, and B. Zhang, The search for heavy Majorana neutrinos, J. High Energy Phys. 05 (2009) 030.

[10] A. B. Balantekin, A. de Gouva, and B. Kayser, Addressing the Majorana vs Dirac question with neutrino decays, Phys. Lett. B 789, 488 (2019).

[11] P. Ballett, T. Boschi, and S. Pascoli, Heavy neutral leptons from low-scale seesaws at the DUNE near detector, arXiv:1905.00284 [J. High Energy Phys. (to be published)].

[12] R. E. Shrock, New tests for, and bounds on, neutrino masses and lepton mixing, Phys. Lett. 96B, 159 (1980).

[13] R. E. Shrock, General theory of weak leptonic and semileptonic decays. 1. Leptonic pseudoscalar meson decays, with associated tests for, and bounds on, neutrino masses and lepton mixing, Phys. Rev. D 24, 1232 (1981).

[14] P. Ballett, S. Pascoli, and M. Ross-Lonergan, MeV-scale sterile neutrino decays at the Fermilab Short-Baseline Neutrino program, J. High Energy Phys. 04 (2017) 102.

[15] R. Acciarri et al. (MicroBooNE Collaboration), Noise characterization and filtering in the MicroBooNE liquid argon TPC, J. Instrum. 12, P08003 (2017).

[16] J. S. Marshall and M. A. Thomson, The Pandora software development kit for pattern recognition, Eur. Phys. J. C 75, 439 (2015), we use Pandora v03_00_00.

[17] R. Acciarri et al. (MicroBooNE Collaboration), The Pandora multi-algorithm approach to automated pattern recognition of cosmic-ray muon and neutrino events in the MicroBooNE detector, Eur. Phys. J. C 78, 82 (2018).

[18] C. Adams et al. (MicroBooNE Collaboration), Calibration of the charge and energy response of the MicroBooNE liquid argon time projection chamber using muons and protons, arXiv:1907.11736 [J. Instrum. (to be published)].

[19] E. L. Snider and G. Petrillo, LArSoft: Toolkit for simulation, reconstruction and analysis of liquid argon TPC neutrino detectors, J. Phys. Conf. Ser. 898, 042057 (2017).

[20] C. Andreopoulos et al., The GENIE neutrino Monte Carlo generator: Physics and user manual, arXiv:1510.05494, We use GENIE v2.8.6d.

[21] D. Heck et al., CORSIKA: A Monte Carlo code to simulate extensive air showers, FZKA-6019 (Forschungszentrum Karlsruhe, Karlsruhe, 1998).

[22] S. Agostinelli et al. (GEANT4 Collaboration), GEANT4: A simulation toolkit, Nucl. Instrum. Methods Phys. Res., Sect. A 506, 250 (2003), we use GEANT4 v4_10_1_p03d.

[23] J. Allison et al., Recent developments in GEANT4, Nucl. Instrum. Methods Phys. Res., Sect. A 835, 186 (2016).

[24] T. Chen and C. Guestrin, XGBoost: A scalable tree boosting system, in Proceedings of the 22nd ACM SIGKDD International Conference on Knowledge Discovery and Data Mining, KDD '16 (ACM, New York, New York, 2016), pp. 785-794.

[25] The MicroBooNE Collaboration, Booster neutrino flux prediction at MicroBooNE, Report No. MicroBooNENote-1031-PUB, 2018.

[26] C. Adams et al. (MicroBooNE Collaboration), Ionization electron signal processing in single phase LArTPCs. Part I. Algorithm description and quantitative evaluation with MicroBooNE simulation, J. Instrum. 13, P07006 (2018).

[27] C. Adams et al. (MicroBooNE Collaboration), Ionization electron signal processing in single phase LArTPCs. Part II. Data/simulation comparison and performance in MicroBooNE, J. Instrum. 13, P07007, 2018.

[28] T. Junk, Confidence level computation for combining searches with small statistics, Nucl. Instrum. Methods Phys. Res., Sect. A 434, 435 (1999).

[29] A. L. Read, Presentation of search results: The $\mathrm{CL}_{s}$ technique, J. Phys. G 28, 2693 (2002).

[30] W. Fisher, Systematics and limit calculations, Report No. Fermilab-TM-2386-E, 2006.

[31] E. Cortina Gil et al. (NA62 Collaboration), Search for heavy neutral lepton production in $K^{+}$decays, Phys. Lett. B 778, 137 (2018).

[32] A. Vaitaitis et al. (NuTeV Collaboration), Search for Neutral Heavy Leptons in a High-Energy Neutrino Beam, Phys. Rev. Lett. 83, 4943 (1999).

[33] A. V. Artamonov et al. (E949 Collaboration), Search for heavy neutrinos in $K^{+} \rightarrow \mu^{+} \nu_{H}$ decays, Phys. Rev. D 91, 052001 (2015); Erratum, Phys. Rev. D 91, 059903 (2015).

[34] G. Bernardi et al., Search for neutrino decay, Phys. Lett. B 166, 479 (1986).

[35] G. Bernardi et al., Further limits on heavy neutrino couplings, Phys. Lett. B 203, 332 (1988).

[36] K. Abe et al. (T2K Collaboration), Search for heavy neutrinos with the T2K near detector ND280, Phys. Rev. D 100, 052006 (2019). 